Running Head:

WOOD CHAR-WATER SORPTION COEFFICIENTS OF ORGANIC SORBATES

Corresponding Author:

Prof. Desiree L. Plata

Yale University

Department of Chemical and Environmental Engineering

9 Hillhouse Ave

PO Box 208286

New Haven CT 06520

203-436-9066 (T)

203-432-4387 (F)

desiree.plata@yale.edu 


\title{
Polyparameter Linear Free Energy Relationship for Wood Char-Water Sorption Coefficients of Organic Sorbates
}

\author{
Desiree L. Plata ${ }^{* \S}$, Jordon D. Hemingway ${ }^{\ddagger \S}$, and Philip M. Gschwend ${ }^{\S}$ \\ ${ }^{\dagger}$ Department of Chemical and Environmental Engineering, Yale University, New Haven, \\ CT, USA \\ ${ }^{\ddagger}$ Department of Marine Chemistry and Geochemistry, Woods Hole Oceanographic \\ Institution, Woods Hole, MA, USA \\ ${ }^{\S}$ Department of Civil and Environmental Engineering, Massachusetts Institute of \\ Technology, Cambridge, MA, USA
}


WOOD CHAR-WATER SORPTION COEFFICIENTS OF ORGANIC SORBATES

*Address correspondence to desiree.plata@yale.edu 
WOOD CHAR-WATER SORPTION COEFFICIENTS OF ORGANIC SORBATES

\begin{abstract}
Black carbons (BCs), including soots, chars, activated carbons, and engineered nanocarbons, have different surface properties, but we do not know to what extent these affect their sorbent properties. To evaluate this for an environmentally ubiquitous form of BC, biomass char, we probed the surface of a well-studied wood char using 14 sorbates exhibiting diverse functional groups and then fit the data with a polyparameter linear free energy relationship (ppLFER) to assess the importance of the various possible sorbatechar surface interactions. Sorption from water to water-wet char evolved with the sorbate's degree of surface saturation and depended on only a few sorbate parameters:
\end{abstract}

$\log K_{d}(\mathrm{~L} / \mathrm{kg})=$

$$
\left.\left.\left[(4.03 \pm 0.14)+(-0.15 \pm 0.04) \log a_{i}\right)\right] V+\left[(-0.28 \pm 0.04) \log a_{i}\right)\right] S+(-5.20 \pm 0.21) B
$$

where $a_{i}$ is the aqueous saturation of the sorbate $i, V$ is McGowan's characteristic volume, $S$ reflects polarity, and $B$ represents the electron-donation basicity. As generally observed for activated carbon, the sorbate's size encouraged sorption from water to the char, while its electron donation/proton acceptance discouraged sorption from water. However, the magnitude and saturation dependence differed significantly from what has been seen for activated carbons, presumably reflecting the unique surface chemistries of these two BC materials and suggesting BC-specific sorption coefficients will yield more accurate assessments of contaminant mobility and bioavailability and evaluation of a site's response to remediation. 
WOOD CHAR-WATER SORPTION COEFFICIENTS OF ORGANIC SORBATES

\section{Keywords}

Sorption, Wood char, Hydrophobic organic compounds (HOCs), Polyparameter linear

free energy relationship (ppLFER), Black carbon (BC) 


\section{WOOD CHAR-WATER SORPTION COEFFICIENTS OF ORGANIC SORBATES}

\section{INTRODUCTION}

In an effort to manage contaminated sediments and soils, regulators try to use data

4 on solid phase concentrations of organic contaminants to identify deposits that pose

5 unacceptable toxic risks. Commonly, this is done by assuming equilibrium partitioning

6 of the compounds among the solid phases, porewater or soil gas, and associated

7 organisms [1]. The key parameter allowing us to use sediment/soil concentrations in this

8 model is the solid-water sorption coefficient, $K_{d}\left[\left(\mathrm{~mol} / \mathrm{kg}_{\text {solid }}\right) /\left(\mathrm{mol} / \mathrm{L}_{\text {water }}\right)\right]$. In the case of

9 contaminated sediments, this allows us to estimate porewater concentrations, $\mathrm{C}_{\mathrm{w}}$, from

10 the ratio, $\mathrm{C}_{\text {sed }} / K_{d}$.

11 Until now, regulators have assumed that $K_{d}$ for most nonionic contaminants is

12 given by the product, $\mathrm{f}_{\mathrm{oc}} K_{o c}$, where $\mathrm{f}_{\mathrm{oc}}$ is the organic carbon content of the sediment

$13\left(\mathrm{~kg}_{o c} / \mathrm{kg}_{\text {solid }}\right)$ and $K_{o c}$ is the organic carbon-normalized solid-water partition coefficient

$14\left[\left(\mathrm{~mol} / \mathrm{kg}_{\mathrm{oc}}\right) /\left(\mathrm{mol} / \mathrm{L}_{\text {water }}\right)\right]$. However, such sorption modeling has been found to be

15 extremely inaccurate at many sites [2,3]. If it were accurate, then one should find close

16 correspondence between porewater concentrations and sediment concentrations

17 normalized by $\mathrm{f}_{\mathrm{oc}} K_{o c}$. Instead, measured values of $\mathrm{C}_{\mathrm{w}}$ are commonly up to a factor of 100

18 less than $\mathrm{C}_{\mathrm{sed}} / \mathrm{f}_{\mathrm{oc}} K_{o c}$ [4]! Likewise, biota-sediment accumulation factors (BSAFs)

19 measured for a wide range of benthic infauna are often 1 to 2 orders of magnitude below

20 the corresponding equilibrium partitioning expectation [5-7]. This situation suggests that

21 there is a major problem with respect to estimating solid-water partition coefficients

22 using only an $\mathrm{f}_{\mathrm{oc}} K_{o c}$ model. 


\section{WOOD CHAR-WATER SORPTION COEFFICIENTS OF ORGANIC SORBATES}

We now know that sediments and soils include "black carbons" (BCs) such as

24 soots, biomass chars, and coal dust residues, and that these special components of the

25 total organic carbon must be distinguished from the rest of the organic matter to estimate

26 accurately $K_{d}$ values of many contaminants [8-14]. Thus, sorption estimations can be

27 greatly improved by using an extended expression that includes black carbon as a

28 separate sorbent [10]

$$
K_{d}=\mathrm{f}_{\mathrm{oc}} K_{o c}+\mathrm{f}_{\mathrm{BC}} K_{B C} \mathrm{C}_{\mathrm{w}}^{(n-1)}
$$

32 where $f_{o c}$ is now the non- $B C$ organic carbon weight fraction in the sediment, $f_{B C}$ is the

33 BC weight fraction, $K_{B C}$ is the compound specific BC-water adsorption coefficient, and

$34 \mathrm{C}_{\mathrm{w}}{ }^{(n-\mathrm{l})}$ is the dissolved sorbate concentration of compound raised to a power, $n-1$, to

35 reflect the nonlinearity of adsorption to the BC surface. To date, the majority of available

$36 K_{B C}$ values are derived from studies using engineered, granular activated carbon (GAC)

37 as a sorbent, but distinct BCs may have different sorption coefficients from GAC. If so,

38 then solid-water partitioning models would be improved by the use of the appropriate $K_{B C}$

39 when the sorbent identity is known (e.g., near a coal storage site or after application of an

40 engineered biochar). In the present study, we explore the sorption parameters for a

41 biochar ( $K_{\text {char }}$ and $n_{\text {char }}$ ) for a structurally diverse array of sorbates and compare those to

42 sorption parameters for GAC in order to determine if BC-specific sorption parameters are

43 needed.

44 Chars, themselves, are ubiquitous and structurally diverse [15] and can have a 45 wide range of surface areas and surface functionalities, depending on the conditions of 
WOOD CHAR-WATER SORPTION COEFFICIENTS OF ORGANIC SORBATES

46 their formation [16, 17]. However, chars formed at greater than about $400^{\circ} \mathrm{C}$ typically

47 have predominantly aromatic surface character [17-19] and some oxygen-containing

48 functionalities [20, 21]. Further, biochars are increasingly investigated as "green”

49 sorbates, as they can be made from waste biomaterials, and they have abundant natural,

50 incidental, and engineered sources globally (Figure 1).

51 Hence, here we characterize sorption of 14 diverse sorbates to a wood char

52 formed at $450^{\circ} \mathrm{C}$. After determining the isotherms, polyparameter linear free energy

53 relationship (ppLFER) modeling was used to identify the most important intermolecular

54 interactions controlling sorption to this water-wet char. The choice of model sorbates

55 was intended to capture the widest degree of variability in the Abraham/McGowan [25,

5626 ] sorbate parameter space with a relatively small number of sorbates that are liquids at

57 room temperature. Overall, our goals were to (a) enable prediction of partitioning

58 coefficients for untested compounds whose parameters fall within our tested range for

59 this particular wood char and (b) add to the understanding of the fundamental

60 mechanisms of sorption from water to unique biochars. We note that applying the

61 resultant ppLFER outside the training set parameter space (e.g., extrapolating to estimate

$62 \mathrm{~K}_{\mathrm{BC}}$ values for PCBs) may result in large errors in estimated $K_{d}$ s, especially as steric

63 issues related to the sorbates' surface interactions and pore access can arise.

64

65

EXPERIMENTAL METHODS

66

67 Chemical sorbates. 


\section{WOOD CHAR-WATER SORPTION COEFFICIENTS OF ORGANIC SORBATES}

We used 14 sorbates (Table 1) with varied functional groups, covering a range of

69 the ppLFER parameter space (i.e., $V$ from 0.715 to $1.154, E$ from 0 to $0.871, S$ from 0 to

70 1.11, $A$ from 0 to 0.37 , and $B$ from 0 to 0.51 ). While these probe compounds do not

71 cover the entire known range of the parameters [25, 26], they do represent diverse

72 intermolecular forces and have a relatively broad range in aqueous solubilities (Table 1).

73 Any ppLFER can only be justifiably applied within the tested Abraham/McGowan

74 parameter space, rather than outside of it (i.e., the V, E, S, A, and B of a compound must

75 fall within the range tested here if the ppLFER is to give accurate results). Sorbate-

76 saturated aqueous solutions were prepared by equilibrating excess quantities of each

77 sorbate with $18 \mathrm{M} \Omega \mathrm{UV}$-oxidized water for at least $1 \mathrm{wk}$ in pre-combusted, ground-glass-

78 stoppered flasks.

80 Char sorbent.

81 Wood char, prepared from chestnut hardwood pyrolyzed at $450^{\circ} \mathrm{C}$ as described in

82 Hammes et al. [21; see also 27, 28], was purchased from the Schmidt Laboratory at the

83 University of Zurich (Supplemental Data, Table S1). To ensure uniform sorbate

84 exposure to char surfaces and ultimately aid in gravitational settling of the char, the fine

85 char particles were dispersed in ethyl acetate, spread over pre-combusted, acid-washed,

86 Ottawa sand, and then stirred semi-continuously while air drying. The char-quartz

87 mixture was further dried at $60^{\circ} \mathrm{C}$ for $72 \mathrm{~h}$, with stirring every $12 \mathrm{~h}$. The weight percent

88 carbon contents of the quartz, char, and the char-quartz mixture were $0.016 \pm 0.006,71.7$

$89 \pm 0.2$, and $1.1 \pm 0.2 \% \mathrm{C}$, respectively, as determined using a CHN analyzer (Vario EL,

90 Elementar America, Inc.) operated at a combustion temperature of $950{ }^{\circ} \mathrm{C}$. Specific areas 


\section{WOOD CHAR-WATER SORPTION COEFFICIENTS OF ORGANIC SORBATES}

91 for quartz, char, and the char-quartz mixture were 3.3, 5.9, and $3.6 \mathrm{~m}^{2} \mathrm{~g}^{-1}$, respectively, as

92 determined by single-point nitrogen BET (Brunauer-Emmett-Teller) testing (PMI

93 Analytical Testing, Ithaca, NY). Note that Hammes et al. [21] reported $2.0 \mathrm{~m}^{2} \mathrm{~g}^{-1}$ for this

94 particular wood char.

95

96 Sorption equilibration experiments.

97 All batch sorption experiments were conducted in pre-combusted, 7-mL, 18-mL,

98 or 50-mL ground-glass-stoppered centrifuge tubes. All-glass containers were used to

99 avoid sorbate losses to Teflon ${ }^{\mathrm{TM}}$ or other polymers [29-31]. Sorbent (i.e., char-quartz

100 mixture) and sorbent-free (i.e., quartz only) samples were pre-wet with $18 \mathrm{M} \Omega$ water for

101 at least 1 wk prior to sorbate addition. Serial dilutions of the water-saturated sorbate

102 stocks were gravimetrically prepared (at $0.1,0.01,0.001$, and 0.0001 aqueous saturation)

103 and then immediately added to triplicate sorbent containers. Headspace was minimized

104 to avoid sorbate loss to the vapor phase. Sorbate concentrations were quantified

105 following 7-to-14 d equilibration times, which we determined was sufficient to establish

106 equilibrium (based on a toluene sorption time-series (Supplemental Data, Figure S1;

107 Table S2). To minimize the influence of suspended colloids on the measured dissolved

108 aqueous concentrations of sorbate, each test tube was centrifuged at $1150 \mathrm{~g}$ for at least 90

109 min prior to sorbate quantification. Recall that the char itself was spread over quartz sand

110 (through the use of an ethyl acetate carrier) to ensure uniform exposure of the char and to

111 aid gravitational settling.

112 As sorption to char is a surface process, we chose to probe the char surface as a

113 function of sorbate activities, rather than concentrations. Here, recognizing that these 


\section{WOOD CHAR-WATER SORPTION COEFFICIENTS OF ORGANIC SORBATES}

114 liquid sorbate's solubilities represent $100 \%$ saturation in solution, the fractional saturation

115 in solution also corresponds to the same degree of saturation (or chemical activity) on the

116 char surface at solution-surface equilibrium in a batch experiment. This allowed us to

117 investigate the effect of increasing surface coverage between highly variable sorbates.

118 Since the probe sorbates' have a broad range of aqueous solubilities, and comparison at

119 equal concentrations would either unnecessarily limit the range of chemical loadings (i.e.,

120 for the most water-soluble species) or result in the formation of a pure sorbate phase (i.e.,

121 for the least soluble species), which would confound the results.

122

123 Sorbate quantification and sorption calculation.

124 Analytes with relatively low aqueous solubilities $(<6,000 \mathrm{mg} / \mathrm{L})$ were quantified

125 using a purge and trap pre-concentrator (Tekmar LCS 2000) interfaced to a gas

126 chromatograph (GC; Perkin Elmer AutoSystem XL) with a flame ionization detector

127 (FID) for hydrocarbons or an electron capture detector (ECD) for halogenated

128 compounds. Analytes with relatively high aqueous solubilities (>6,000 mg/L) were

129 quantified via direct aqueous injection onto a GC-FID (Carlo Erba HRGC 5300 Mega

130 Series MFG 500). Both instruments were equipped with a DB-624 capillary column (60

$131 \mathrm{~m} \times 3.80 \mathrm{um} \times 0.320 \mathrm{~mm}$ ) and temperature programs varied with the analyte.

132 The average aqueous sorbate concentration in char-free control tubes (i.e., quartz

133 only; $n=3$ ) was compared to the aqueous sorbate concentration in char-containing tubes

134 (i.e., $\mathrm{C}_{\mathrm{w}}$ ), where the difference was attributed to sorption to char. This mass difference

135 was normalized to the mass of char sorbent to give the concentration of sorbate on the

136 solid char (i.e., $\mathrm{C}_{\mathrm{s}}$ ). For hexane and heptane, which have relatively high air-water 
137 partition coefficients, we also accounted for the mass of sorbate in test tube bubbles that

138 evolved during the course of the equilibration $(<1 \mathrm{~mL}$, where the volume was deduced by

139 changes in weight).

141 Sorption isotherms.

142 The experimentally-determined $\mathrm{C}_{\mathrm{s}}$ and $\mathrm{C}_{\mathrm{w}}$ data were fit to the log-transformed

143 Freundlich equation

$$
\log C_{s}=\log K_{f}+n \log C_{w}
$$

147 where $C_{s}$ and $C_{w}$ are equilibrium concentrations in the solid phase $(\mathrm{mg} / \mathrm{kg})$ and aqueous

148 phase $(\mathrm{mg} / \mathrm{L})$, respectively, and $K_{f}\left[(\mathrm{mg} / \mathrm{kg}) /(\mathrm{mg} / \mathrm{L})^{n}\right]$ and $n$ (dimensionless) are the

149 Freundlich coefficient and Freundlich exponent, respectively.

150

151 Polyparameter linear free energy relationship.

152 All measures of $K_{d}$ (i.e., each $\mathrm{C}_{\mathrm{w}} / \mathrm{C}_{\mathrm{s}}$ pair) were used to evaluate the dependence

153 of $K_{d}$ on sorbate properties (i.e., the Abraham/McGowan parameters) by fitting the

154 saturation-dependent ppLFER (Eqn. 3).

$155 \log K_{d, a c t i v i t y}(\mathrm{~L} / \mathrm{kg})=\left(v_{1}+v_{2} \log a_{i}\right) V+\left(e_{1}+e_{2} \log a_{i}\right) E+\left(s_{1}+s_{2} \log a_{i}\right) S$

$156+\left(a_{1}+a_{2} \log a_{i}\right) A+\left(b_{1}+b_{2} \log a_{i}\right) B+c_{1}+c_{2} \log a_{i}$

157 where $V\left(\right.$ in cm $\left.^{3} \mathrm{~mol}^{-1} / 100\right)$ is the sorbate's McGowan characteristic volume, $E$ (in

$158 \mathrm{~cm}^{3} / 10$ ) is the excess molar refraction, $S$ is the polarity/polarizability parameter, $A$ and $B$

159 are the hydrogen acidity and basicity, respectively, and $a_{i}=C_{i w} / C_{i w, s a t}$, is the ratio of the 
160 sorbate i's concentration to its (liquid) solubility. Coefficients were determined both by

161 backward and forward stepwise multiple linear regression analysis of experimental values

162 using Microsoft ${ }^{\mathrm{TM}}$ Excel and StatPlus ${ }^{\mathrm{TM}}$. In the case of backward fitting, the significance

163 of each term in equation 3 was evaluated using a $t$ test to determine the probability that

164 the coefficient was different from zero. Insignificant terms were systematically

165 eliminated one at a time and then the regression analysis was repeated until only

166 significant correlation coefficients remained (i.e., via backward stepwise multiple

167 regression analysis) and the ANOVA factor, F, was maximized. Given a data set of 128

168 observations, 6-12 parameters could be fit meaningfully, but we ultimately found only 4

169 parameters proved to be significantly different from zero in the optimized ppLFER fit.

170 For comparison, a forward stepwise multiple linear regression analysis yielded the same

171 significant terms and coefficients (Supplemental Data, Table S3).

\section{RESULTS}

175 Sorption kinetics.

176 Sorption time courses were assessed using toluene, which has a similar aqueous

177 diffusivity (ca. $10^{-5} \mathrm{~cm}^{2} \mathrm{~s}^{-1}$ ) as our other probe sorbates. Dissolved toluene

178 concentrations approached equilibrium in less than 1 week (Supplemental Data, Figure

179 S1), and thus, we concluded that our incubation timeframe should be at least $1 \mathrm{wk}$ to

180 establish equilibrium, while at the same time not suffering losses during the incubations

181 (e.g., since bubbles appeared). More sorptive probe compounds, such as pyrene or

182 polychlorinated biphenyls, experience even greater retardation of diffusive transport into 
WOOD CHAR-WATER SORPTION COEFFICIENTS OF ORGANIC SORBATES

183 porous char. Thus, they require longer equilibration times and are not yet included in this

184 set of probe compounds.

185

186 Sorption isotherms.

187 The Freundlich equation (Eqn. 1) fit the data for each sorbate well, with

188 correlation coefficients of at least 0.96 , except for heptane $\left(R^{2}=0.77\right)$ and diisopropyl

189 ether $\left(R^{2}=0.87\right.$; Figure 2 and Table 2$)$. Each isotherm was constructed using at least

190 eight data points, except for 1-heptanol $(N=6)$. Within each compound class (e.g.,

191 alkanes, ethers, and alcohols), sorbates with the highest aqueous solubility exhibited the

192 lowest affinity for char (lowest sorption), except for diethylether (Figure 2). For alkanes,

193 alcohols, and ketones, the seven-carbon sorbate always had a larger $K_{f}$ than the respective

194 six-carbon compounds (Table 2), likely reflecting their increased van der Waals

195 interactions with the char surface and increased cost of cavity formation in water due to

196 the addition of methylene groups. Although diethyl ether was the most soluble ether,

197 implying greater relative affinity for the solution phase, its larger $K_{f}$ value compared to

198 MTBE and diisopropyl ether suggested intermolecular interactions with the char that

199 were not applicable to the branched ethers, such as due to steric effects.

200

201 Polyparameter linear free energy relationships.

202 To capture the saturation-dependent behavior of sorption, we fit the expanded

203 LFER expression (Eqn. 3), which includes a dependence on aqueous saturation ( $a=$

$\left.204 \mathrm{C}_{\mathrm{w}} / \mathrm{C}_{\mathrm{w}, \text { sat }}\right)$, noting that aqueous saturation and sorption saturation must occur together in

205 our experiment if we successfully achieved equilibration. Using the 128 independent 


\section{WOOD CHAR-WATER SORPTION COEFFICIENTS OF ORGANIC SORBATES}

$206 K_{d, a c t i v i t y}$ observations (Figure 3), we optimized the fit via the multiple regression statistic

$207 \quad$ F and found

208

$\left.209 \log K_{d, a c t i v i t y}(\mathrm{~L} / \mathrm{kg})=[(4.03 \pm 0.14)+(-0.15 \pm 0.04) \log a)\right] V$

210

211

$$
+[(-0.28 \pm 0.04) \log a)] S+(-5.20 \pm 0.21) B
$$

$$
\mathrm{R}^{2}=0.98, \mathrm{~N}=128, \mathrm{SE}=0.41
$$

215 For our data set, the coefficients for the $A$ (hydrogen acidity/electron accepting capacity)

216 and $E$ (molar refraction/polarizability) terms were not significantly different from zero,

217 suggesting these parameters did not substantially influence char-water sorption. Note

218 that the tested range of A was comparatively small (0 to 0.37), and dependence on A may

219 emerge if the data set were expanded. Further, the ppLFER should only be employed for

220 sorbates whose Abraham/McGowan parameters fall within the tested parameter space

221 (Table 1). Char-water partitioning depended directly on the dispersion interactions ( $V$

222 term), inversely on the electron donating character ( $B$ term), and weakly on the sorbate’s

223 polarizability ( $S$ term). Interestingly, the $V$ term exhibited some dependence on

224 saturation, becoming more important at higher dilution; the $B$ term did not exhibit

225 significant dependence on saturation over the investigated range, and the $S$ term was only

226 important at low activities (e.g., $a<0.001$ ). The physical-chemical implications of these

227 dependencies are discussed below. 


\section{WOOD CHAR-WATER SORPTION COEFFICIENTS OF ORGANIC SORBATES}

\section{DISCUSSION}

231 Consistency of ppLFER with previous observations.

232 Due to the prevalent use of activated carbon in water remediation applications,

233 several research groups have previously developed activated carbon-water partitioning

234 LFERs for organic compounds. While the exact coefficients differ (Table 3) [32-36],

235 likely due to differences in GAC character and perhaps some experimental problems

236 associated with assuming equilibrium before it is reached (Supplemental Data, Table S2),

237 the important sorbate parameters are consistent: the molar volume $(V)$ and electron-

238 donation basicity $(B)$ always exhibit the largest influence on the distribution coefficient,

$239 K_{d}$. The dispersion term ( $V$ ) always encourages sorption and the electron-donating

240 basicity $(B)$ of a sorbate always reduced sorption, and both were clearly different from

241 zero.

242 The positive coefficient on the $V$ term (Eqn. 4) largely results from the favorable

243 free energy change associated from moving out of water to a hydrophobic surface. The

244 activity dependency suggests more complete sorbate removal at low saturations

245 (increasing influence of $V$ term at low surface coverage), consistent with the view that the

246 most favorable sorption sites involve deeper sorbate penetration into the porous char.

247 The negative impact of the $B$ term implies that loss of hydrogen-bond donating 248 interactions between the sorbate and solvent water are not replaced by interactions with

249 the water-wet char. This is consistent with the expectation that aromatic pi electrons in 250 the char can donate electron density to a hydrogen-bonding sorbate but cannot accept 251 electron density from electron-donating sorbates [37]. The absence of aqueous saturation 


\section{WOOD CHAR-WATER SORPTION COEFFICIENTS OF ORGANIC SORBATES}

252 dependence of the $B$ term for char sorption suggests that this effect is independent of the

253 surface coverage on the char. In other words, the saturation independent character and

254 negative coefficient of B may simply reflect a constant "penalty” of sorbate removal from

255 water, irrespective of sorbate position within the char.

256 Other terms made smaller contributions to $K_{d}$, as was observed for activated

257 carbon (see Table 3). In the case of the wood char, the contribution of the polarizability

258 parameter $(S)$ to $K_{d}$ was weak but activity dependent, becoming more positive at lower

259 sorbate coverage. This may imply that dipole-induced dipole or dipole-dipole

260 interactions with char are stronger at lower char surface coverages. At higher degrees of

261 saturation, the dipole-induced dipole and dipole-dipole interactions with water and water-

262 wet char may be roughly equivalent, and thus, the effect of the $\mathrm{S}$ term decreases in 263 significance.

Interpretation of Freundlich parameters.

266 The Freundlich isotherm parameters $K_{f}$ and $n$ are influenced by a sorbate's

267 structural parameters, and the ppLFER's dependence on chemical aqueous saturation

268 allowed us to investigate this relationship. The distribution coefficient, $K_{d}$, is equal to

$269 C_{s} / C_{w}$, and chemical activity, $a$, expresses the saturation level, $C_{w} / C_{w, s a t}$; substituting

270 these ratios into Equation 4 and rearranging yields

271

$272 \log C_{s}=(4.03 V-5.20 \mathrm{~B})+(0.15 V+0.28 \mathrm{~S}) \log C_{w, \mathrm{sat}}+$

273

$$
(-0.15 V-0.28 S+1) \log C_{w}
$$


WOOD CHAR-WATER SORPTION COEFFICIENTS OF ORGANIC SORBATES

276 This equation (Eqn. 5) has the form of the log-transformed Freundlich isotherm

277 expression (Eqn. 1), $\log C_{s}=\log K_{f}+n \log C_{w}$, and can thus be solved to determine $\log$

$278 K_{f}$ and $n$ in terms of the sorbate parameters, $V, S$, and $B$.

279

$280 \log K_{f}\left[(\mathrm{mg} / \mathrm{kg}) /(\mathrm{mg} / \mathrm{L})^{n}\right]=(4.03 V-5.20 B)+(0.15 V+0.28 S) \log C_{w, s a t}$

281

282 and

283

$284 n=-0.15 V-0.28 S+1$

286 The accuracy of this approach is suggested by the goodness of fit of the observed data 287 with those predicted by Equations 6 and 7 (Figure 4). As was observed for granular 288 activated carbon (GAC) [32], the $V$ and $B$ terms are also the major contributors to the 289 Freundlich coefficient observed for char. Dispersive forces (captured by the $V$ term)

290 encourage sorption and increase $K_{f}$, whereas electron donation capacity (reflected by the

$291 B$ term) decreases $K_{f}$. A sorbate’s polarizability (S term) makes a minor contribution to

292 the Freundlich coefficient and that contribution is higher for solutes with larger aqueous

293 solubilities. For example, a typical hydrogen-bond accepting sorbate could have $V \approx 1$,

$294 B \approx 0.5, S \approx 0.5$, and $\log C_{w, s a t} \approx \log (1 \mathrm{mg} / \mathrm{L})=1$, and thus, $\log K_{f} \approx 4.18-0.26+0.14 \approx 4$,

295 where the terms correspond to the contributions of $V, B$, and $S$, respectively. In contrast,

296 sorbates with little electron donating basicity and polarizability (i.e., $\mathrm{B} \approx 0$ and $\mathrm{S} \approx 0$ ) have 


\section{WOOD CHAR-WATER SORPTION COEFFICIENTS OF ORGANIC SORBATES}

297 Freundlich coefficients almost entirely dependent on dispersive forces (the $V$ term). This

298 is, perhaps, unsurprising and is consistent with observations.

299 The Freundlich exponent, $n$, captures the non-linearity of the sorption process,

300 and, in the case of sorption from water to water-wet char, this exponent exhibited a

301 dependence on dispersive forces and polarity (i.e., the $V$ and $S$ terms, respectively). Note

302 that the slope of the line is not significantly different from $1(1.19 \pm 0.36)$, indicating that

303 Equation 7 is capturing the right dependencies of $n$ on sorbate properties, but the low $\mathrm{R}^{2}$

304 (0.47) suggests the correlation does not perform with much precision). The $S$ parameter

305 has been correlated with the Freundlich exponent in studies of sorption of organic

306 chemicals to peat soil and GAC [32, 38]. For GAC (Darco 20-40 mesh from Sigma-

307 Aldrich), Shih and Gschwend [32] noted that the electron donating basicity ( $B$ term)

308 made a small contribution to the Freundlich exponent, but this was not observed for our

309 char perhaps due to differences in the oxygen functionality of the materials. For nonpolar

310 compounds (e.g., with $\mathrm{S} \approx 0$ ), the dispersive forces dominate the sorption behavior. For a

311 typical polar sorbate (e.g., with $\mathrm{V} \approx 1$ and $\mathrm{S} \approx 0.5$ ), the Freundlich exponent will have

312 roughly equal contributions from the dispersion and polarity terms, but these will be

313 dominated by the constant (+1). We note that the constant in the $n$ expression for our

314 char $(+1)$ was greater than seen previously for GAC $(+0.76)$ [32]; this could imply that

315 the isotherm nonlinearity also has some dependency on sorbent properties (e.g., GAC vs.

316 wood char). For example, the exponent could capture the chemical nature of the char

317 surface and physical distribution of pore spaces. The Freundlich exponents for char were

318 systematically higher than those for GAC [32] (Supplemental Data, Table S2) and, as

319 noted earlier, could not be predicted by the same LFER. Knappe’s group has 
WOOD CHAR-WATER SORPTION COEFFICIENTS OF ORGANIC SORBATES

320 demonstrated that pore structure and surface composition (e.g., oxygen content) have a

321 significant influence on the sorption behavior of organic chemicals [39,40], and

322 differences in the physicochemical profile of the sorption surface may explain apparent

323 differences in sorption properties of distinct BCs. Thus, sorbent-specific free energy

324 relationships may be needed for distinct types of black carbon sorbent materials.

\section{CONCLUSIONS}

328 Overall, three primary lines of evidence support the results presented here and

329 build confidence in this sorbent characterization approach: (1) the Freundlich parameters,

$330 K_{f}$ and $n$, individually make sense (e.g., polar compounds have $n$ values closer to 1 and

331 hydrophobic sorbates have larger $K_{f}$ ); (2) the ppLFER yielded four best-fit parameters

332 (i.e., $v_{1}, v_{2}, b_{1}$, and $s_{2}$ in Eqns. 3 and 4) that were significantly different from zero from

333128 individual observations; and (3) previous researchers have also observed positive

334 relationships between $K_{d}$ and $V$ and negative relationships between $K_{d}$ and B for similar

335 forms of black carbon. Further, the fit also indicates that considering sorption as a

336 function of the degree of surface saturation (where complete saturation is assumed to

337 correspond to saturation in solution) yields a ppLFER that is understandable with respect 338 to sorbate-sorbent interactions.

339 Moreover, our results support the hypothesis that two distinct types of black

340 carbon, GAC and wood char, interact with organic sorbates via subtly different

341 combinations of intermolecular interactions, which arise from the physical and chemical

342 character of the respective sorbent materials. This implies a need for sorbent-specific 
WOOD CHAR-WATER SORPTION COEFFICIENTS OF ORGANIC SORBATES

343 black-carbon distribution coefficients in order to predict contaminant distribution in

344 natural sediments or amended cleanup sites. Fortunately, we found that a relatively small

345 set of sorbate probe compounds can be used to generate a ppLFER that enables

346 estimation of BC-water distribution coefficients for sorbates of interest where sorption

347 data are unavailable (with the caution that the ppLFER should be used within the

348 investigated Abraham/McGowan parameter space (Table 1)). Further work needs to be

349 done to extend the tested parameter space, being sure to utilize sufficient incubation times

350 to insure solution-sorbent equilibration.

351 Furthermore, natural systems with more than one source of BC may require

352 quantification of each BC type to accurately predict contaminant distribution. While

353 independent measures to quantify distinct BC fractions are available, no single method

354 exists to differentiate all possible fractions of BC in environmental matrices. Such a

355 method would be useful if important chemical differences between BC types are

356 confirmed.

357

358

\section{SUPPLEMENTAL DATA}

359 Wood char characteristics, previously modeled black carbons and their sorbent

360 parameters, toluene equilibration timescale for water-wet char, and comparison of

361 Freundlich exponents between GAC and char.

362

363

\section{ACKNOWLEDGMENT}

364 The authors thank J. MacFarlane, M. Streng, Z. Henrot, and E. Forsley. This material is

365 based upon work supported by the U.S. Army Corps of Engineering, Humphreys 
WOOD CHAR-WATER SORPTION COEFFICIENTS OF ORGANIC SORBATES

366 Engineer Center Support Activity under Contract No. W912HQ-10-C-0005 awarded as

367 part of the SERDP program. The authors do not have any conflicts of interest to disclose.

\section{REFERENCES}

370 1. DiToro, D. M., C. Zarba, Hansen, D. J. , Berry, W. , Swartz, R. C., Cowan, C.,

371 Pavlou, S., Allen, H., Thomas, N., and Paquin, P. 1991. Technical basis for establishing sediment quality criteria for nonionic organic-chemicals using

373 equilibrium partitioning. Environmental Toxicology and Chemistry 10, 1541.

374 2. Hawthorne, S.B., Grabanski, CB, Miller, DJ. 2006. Measured partitioning

375 coefficients for parent and alkyl polycyclics aromatic hydrocarbons in 114

376 historically contaminated sediments: Part 1. K-OC values. Environmental

377 Toxicology and Chemistry 25 (11): 2901-2911.

378 3. Hawthrone, SB, Grabanski, CB, Miller DJ. Measured partition coefficients for 379 parent and alkyl polycyclic aromatic hydrocarbons in 114 historically contaminated sediments: part 2. Testing the $\mathrm{k}(\mathrm{oc}) \mathrm{k}(\mathrm{bc})$ two carbon-type model.

382 4. Fernandez, L.A., MacFarlane, J.K., Tcaciuc, A.P., Gschwend, P.M. 2009.

383 Measurement of freely dissolved PAH concentrations in sediment beds using 384 passive sampling with low density polyethylene strips. Environ. Sci. \& 385 Technol., 43, 1430-1436.

$386 \quad$ 5. Tracey, G.A., Hansen D.J. 1996. Use of biota-sediment accumulation factors to 387 assess similarity of nonionic organic chemical exposure to benthically-coupled 


\section{WOOD CHAR-WATER SORPTION COEFFICIENTS OF ORGANIC SORBATES}

475.

390 6. Hellou, J., Steller, S., Zitko, V., Leonard, J., King, T., Milligan, T.G., Yeats, P. 2002. Partitioning of polycyclic aromatic hydrocarbons between water and particles compared to bioaccumulation in mussels: a harbour case. Mar. Env. Res. 53, 357-379.

7. Kraaij, R.H., Tolls, J., Sijm, D., Cornelissen, G., Heikens, A., Belfroid, A. 2002. Effects of contact time on the sequestration and bioavailability of different classes of hydrophobic organic chemicals to benthid oligochaetes (Tubificidae). Environmental Toxicology and Chemistry 21(4), 752-759.

398 8. Gustafsson, O., Haghseta, F., Chan, C., MacFarlane, J.K., Gschwend, P.M. 1997. Quantification of the dilute sedimentary soot phase: Implications for PAH speciation and bioavailability. Environ. Sci. Technol. 31(1), 203-209.

4019 9. Ghosh, U., Gillette, J.S., Luthy, R.G., Zare, R.N. 2000. Microscale location, characterization, and association of polycyclic aromatic hydrocarbons on harbor sediment particles. Environ. Sci. Technol. 34, 1729-1736.

404 10. Accardi-Dey, A., Gschwend P.M. 2002. Assessing the combined roles of 405 natural organic matter and black carbon as sorbents in sediments. Environ. Sci. 406 Technol. 36, 21-29.

407 11. Accardi-Dey, A., Gschwend P.M. 2003. Reinterpreting literature sorption data 408 considering both absorption into organic carbon and adsorption onto black carbon. Environ. Sci. Technol. 37, 99-106. 


\section{WOOD CHAR-WATER SORPTION COEFFICIENTS OF ORGANIC SORBATES}

410 12. Ghosh, U., Zimmerman, J.R., Luthy, R.G. 2003. PCB and PAH speciation among particle types in contaminated sediments and effects on $\mathrm{PAH}$ bioavailability. Environ. Sci. Technol. 37, 2209-2217.

413 13. Lohmann, R., MacFarlane, J.K., Gschwend, P.M. 2005. Importance of black 414 carbon to sorption of native PAHs, PCs, and PCDDs in Boston and New York $415 \quad$ Harbor Sediments. Environ. Sci. Technol. 39, 141-148.

416 14. Ghosh, U., Talley, J.W., Luthy, R.G. 2011. Particle-Scale Investigation of PAH 417 Desorption Kinetics and Thermodynamics from Sediment. Environ. Sci.

$418 \quad$ Technol. 35, 3468-3475.

419 15. McBeath, A.V., Smernik, R.J., Krull, E.S. 2013. A demonstration of the high $420 \quad$ variability of chars produced from wood in bushfires. Organic Geochemistry $421 \quad 55: 38-44$.

422 16. Cao, X., Pignatello, J.J.; Li, Y.; Lattao, C.; Chappell, M.A., Chen, N.A., Miller, 423 L.F., Mao, J. 2012. Characterization of wood chars produced at different 424 temperatures using advanced solid-state ${ }^{13} \mathrm{C}$ NMR spectroscopic techniques. $425 \quad$ Energy \& Fuels 26, 5983-5991.

426 17. Lattao, C., Cao, X., Mao, J., Schmidt-Rohr, K., Pignatello, J.J. 2014. Influence 427 of molecular structure and adsorbent properties on sorption of organic 428 compounds to a temperature series of wood chars. Environ. Sci. Technol. 48, $429 \quad$ 4790-4796.

430 18. Goldberg, E.D. Black Carbon in the Environment, 1985, John Wiley, Hoboken, $431 \quad$ N.J. 


\section{WOOD CHAR-WATER SORPTION COEFFICIENTS OF ORGANIC SORBATES}

432 19. Baldock, J.A.; Smernik, R.J. 2002. Chemical composition and bioavailability

433 of thermally altered Pinus resiinosa (Red pine) wood. Org. Geochem. 33,

434 1093-1109.

435 20. Chun, Y., Sheng, G., Chiou, C.T. 2004. Evaluation of current techniques for

436 isolation of chars as natural adsorbents. Environ. Sci. Technol. 38, 4227-4232.

437 21. Hammes, K., Smernik, R.J., Skjemstad, J.O., Herzog, A., Vogt, U.F., Schmidt, 438 M.W.I. 2006. Synthesis and characterization of laboratory-charred grass straw 439 (Oryza sativa) and chestnut wood (Castanea sativa) as reference materials for $440 \quad$ black carbon quantification. Organic Geochemistry 37, 1629-1633.

441 22. European Environment Agency, Annual European Union Greenhouse Gas

442 Inventory 1990-2012 and Inventory Report, Technical Report No. 9, Annex 2.9

443 CRF Tables LULUCF, European Union, 2014

23. Food and Agricultural Organization of the United Nations, Statistics Division 445 (FAOSTAT), Biomass Burning, All, 1961-2011, Accessed in September 2014 446 via the World Wide Web.

447 24. Jaffe, R., Ding, Y., Niggemann, J., Vahatalo, A. V., Stubbins, A., Spencer, R. 448 G. M., Campbell, J., Dittmar, T. 2013. Global charcoal mobilization from soils 449 via dissolution and riverine transport to the oceans. Science 340 (6130), 345$450 \quad 347$.

451 25. Tulp, H.C., Goss, K-U, Schwarzenbach, R.P., Fenner, K. 2008. Experimental 452 determination of LSER parameters for a set of 76 diverse pesticides and 453 pharmaceuticals. Environ. Sci. Technol. 42, 2034-2040.

454 26. Zissimos, A. M., Abraham, M. H., Kamlet, A., Eclert, F., Wood, J. 2002. A 
WOOD CHAR-WATER SORPTION COEFFICIENTS OF ORGANIC SORBATES

455

456

457

458

460

461

462

463

464

465

466

467

468

469

470

471

472

473

474

475

476

477

Comparison between the two general sets of linear free energy descriptors of

Abraham and Kamlet. J. Chem. Inf. Comput. Sci. 42: 1320-1331.

27. Elmquist, M., G. Cornelissen, Z. Kukulska, Gustafsson, Ö. 2006. Distinct

oxidative stabilities of char versus soot black carbon: Implications for

quantification and environmental recalcitrance. Global Biogeochem. Cycles 20, GB2009, 11 pp.

28. Hammes, K., Schmidt, M.W. I., Smernik, R.J., Currie, L.A., Ball, W.P., Nguyen, T.H., Louchouarn, P., Houel, S., Gustafsson, Ö., Elmquist, M., Cornelissen, G., Skjemstad, J.O., Masiello, C.A., Song, J., Peng, P., Mitra, S., Dunn, J.C., Hatcher, P.G., Hockaday, W.C., Smith, D.M., Hartkopf-Fröder, C., Böhmer, A., Lüer, B., Huebert, B.J., Amelung, W., Brodowski, S., Huang, L., Zhang, W., Gschwend, P.M., Flores-Cervantes, D.X., Largeau, C., Rouzaud, J.-N., Rumpel, C, Guggenberger, G., Kaiser, K., Rodionov, A., Gonzalez-Vila, F.J., Gonzalez-Perez, J.A., de la Rosa, J.M., Manning, D.A.C., López-Capél, E., Ding. L. 2007. Comparison of quantification methods to measure fire-derived (black/elemental) carbon in soils and sediments using reference materials from soil, water, sediment and the atmosphere. Global Biogeochem. Cycles 21 (3): Art. No. GB3016, 18 pp.

29. Lion, L. W., Stauffer, T. B., MacIntyre, W. G. 1990. Sorption of hydrophobic compounds on aquifer materials: Analysis methods and the effect of organic carbon. J. Contam. Hydrol. 5, 215-234.

30. Goss, K.-U., Schwarzenbach, R. P. 1998. Gas/solid and gas/liquid partitioning of organic compounds: critical evaluation of the interpretation of equilibrium 
WOOD CHAR-WATER SORPTION COEFFICIENTS OF ORGANIC SORBATES

constants. Environ. Sci. Technol. 32, 2025-2032.

31. Lung, S. C., Altshul, L. M., Ford, T. E., Spefngler, J. D. 2000. Coating effects on the glass adsorption of polychlorinated biphenyl (PCB) congeners. Chemosphere 41,1865-1873.

32. Kamlet, M. J., Doherty, R. M., Abraham, M H., Taft, R. 1985. Linear solvation energy relationships. XXXIII: An analysis of the factors that influence adsorption of organic compounds on activated carbon. Carbon 23, 549-554.

33. Luehrs, D.C., Hickey, J.P., Nilsen, P.E., Godbole, K.A., Rogers, T.N. 1996a. Linear solvation energy relationship of the limiting partition coefficient of organic solutes between water and activated carbon. Environ. Sci. Technol. 30, 143-152.

34. Luehrs, D.C., Hickey, J.P., Passino-Reader, D.R. 1996b. Linear solvation energy relationships: "Rule of thubs” for estimation of variable values. Environ. Sci. Technol. 30, 143-152.

35. Poole, S. K., Poole, C. F. 1997. Retention of neutral organic compounds from solution on carbon adsorbents. Anal. Commun. 34, 247-251.

36. Shih, Y.-H., Gschwend, P.M. 2009. Evaluating activated carbon-water sorption coefficients of organic compounds using a linear solvation energy relationship (LSER) approach and sorbate chemical activities. Environ. Sci. Technol. 43, 851-857.

37. Schwarzenbach, R.P., Gschwend, P.M., Imboden, D.M. 2003. Environmental Organic Chemistry, 2nd Edition, Wiley-Interscience, New York, N.Y.

38. Endo, S., Grathwohl, P., Haderlein, S. B., Schmidt, T. C. 2008. Compound- 
WOOD CHAR-WATER SORPTION COEFFICIENTS OF ORGANIC SORBATES

501 specific factors influencing sorption nonlineariry in natural organic matter.

502 Environ. Sci. Technol. 42, 5897-5903.

503 39. Li L, Quinlivan, P. A., Knappe, D. R. U. 2002. Effects of activated carbon

504 surface chemistry and pore structure on the adsorption of organic contaminants

505 from aqueous solution. Carbon 40, 2085-2100.

506 40. Li L, Quinlivan, P. A., Knappe, D. R. U. 2005. Predicting adsorption isotherms

507 for aqueous organic micropollutants from activated carbon and pollutant

508 properties. Environ. Sci. Technol. 39, 3393 -3400.

509 
WOOD CHAR-WATER SORPTION COEFFICIENTS OF ORGANIC SORBATES

FIGURE LEGENDS

511 Figure 1. Dominant sources of biochar and illustrated pore and surface structures.

512 Biochar is a ubiquitous sorbate that has natural, incidental, and engineered sources. The

513 arrow thicknesses indicate the postulated relative sizes of the predominant source terms

514 [22-24]. Biochars are formed from the incomplete combustion of biological material,

515 such as wood, leaves, husks, and grasses, and often have microstructures that reflect the

516 structure of the original biological material. (Wood char is depicted here). On the

517 molecular scale, biochar is characterized by a highly aromatic surface with some oxygen

518 content and can interact with sorbents, such as nitrobenzene, via electron donator-

519 acceptor and van der Waals interactions.

520

521 Figure 2. Freundlich isotherms for 14 probe sorbates on chestnut wood char. Solid

522 and dashed lines are best-fit curves, from which we derive characteristic Freundlich

523 parameters, $\log K_{f}$ (intercept) and $n$ (slope). See Table 2 for parameter values.

525 Figure 3. Overall correlation of the individual observed sorption coefficients with

526 the predicted sorption coefficients. Predicted values were determined using the

527 ppLFER given by Equation 4, whereas the observations were experimentally determined

528 from the 128 individual equilibrium experiments conducted in the present study.

530 Figure 4. Correlations of observed Freundlich coefficients, $K_{f}\left[(\mathrm{mg} / \mathrm{kg}) /(\mathrm{mg} / \mathrm{L})^{n}\right]$, and

531 the Freundlich exponent, $\boldsymbol{n}$, with ppLFER-predicted values for those parameters.

532 Predicted values were determined using Equations 6 and 7. Diagonal dashed lines are the 
WOOD CHAR-WATER SORPTION COEFFICIENTS OF ORGANIC SORBATES

533 one-to-one lines, while solid lines are linear best fits. Error bars represent one standard

534 deviation; invisible error bars are smaller than the symbol. 